\title{
THE ANALYSIS OF PREPARATIONS CONTAINING OPIUM.
}

\author{
By Alfred H. Allen and G. E. Scott-Smith.
}

(Read at the Meeting, November 5, 1902.)

OUR attention has been frequently called to the analysis of medicines containing certain preparations of opium, especially paregoric and cough mixtures of various kinds. The analysis of such complex mixtures is by no means simple, and we do not claim to have arrived at a complete method of examination. Paregoric elixir, or compound tincture of camphor of the British Pharmacopcia, is a typical preparation of the kind. It was formerly directed to be prepared from 40 grains each of opium and benzoic acid, 30 grains of camphor, and 30 minims of oil of anise, the whole being diluted with proof spirit to 20 fluid ounces. In the last edition of the British Pharmacopoeia (1898) the opium is replaced by an equivalent amount of tincture of opium, the composition of the preparation remaining otherwise unchanged. The composition of paregoric of commerce varies considerably. The spirit being the most costly ingredient offers an inducement to reduce the proportion present. This practice is objectionable, since the prescribed proportion of oil of anise cannot be kept in solution in a weak spirit. Sometimes only traces of oil of anise are present, in which case the paregoric remains clear when diluted with three or four times its volume of water. The benzoic acid is sometimes deficient in quantity, and is occasionally wholly absent, even in the case of tinctures purchased from registered pharmacists. The opium is the most important remedial constituent of paregoric elixir, and is apt to be deficient in amount or quality, besides being frequently wholly omitted. The last practice is, of course, due to the fact that preparations of opium cannot be legally sold except by registered pharmacists, and hence a preparation destitute of opium is largely substituted by general shopkeepers for the genuine paregoric or compound tincture of camphor of the Pharmacopœia. So-called "paregoric substitutes" and "paregoric without opium " are extensively prepared and sold by shopkeepers, and are even vended by costermongers in the streets of London. Some of these preparations are made simply by omitting the opium from ordinary paregoric. In one instance within our experience the opium of paregoric elixir was replaced by hyoscyamus. Potassium and ammonium bromides are also used in factitious paregoric.

If a measured quantity (25 c.c.) of paregoric be rendered distinctly alkaline with caustic soda, and evaporated to about 10 c.c., the alcohol and a portion of the camphor and oil of anise will be volatilized, and the amount of alcohol can be deduced with sufficient accuracy from the specific gravity of the distillate. On shaking the residual liquid with ether, the remaining camphor and oil of anise will be extracted. If the ether be separated, and the aqueous liquid acidulated with hydrochloric acid, benzoic acid will in some cases be precipitated; but whether it separates or remains in solution it can be dissolved out by agitating the acidified liquid with ether. On allowing the separated ethereal solution to evaporate spontaneously in a small beaker, the benzoic acid is obtained in a state fit to weigh; but a better and more 
rapid plan is to repeatedly agitate the ethereal liquid with water until the washings no longer redden litmus, add a little more water and a few drops of phenolphthalein solution, and titrate the liquid with $\frac{\mathrm{N}}{20}$ caustic alkali (preferably baryta-water), which should be added until the aqueous layer acquires a pink colour, not destroyed by agitation with the ether. Each 1 c.c. of $\frac{N}{20}$ alkali required represents 0.0061 gramme of benzoic acid. If 25 c.c. of the tincture has been employed, the number of milligrammes of benzoic acid found, multiplied by 0.35 , gives the grains of benzoic acid per pint of the tincture. The meconic acid extracted, together with the benzoic acid, is too small in quantity to affect the result, but its presence may be detected and the amount roughly determined by separating the ethereal layer after the titration is complete, and destroying the pink colour of the aqueous liquid by a drop of dilute hydrochloric acid. On now adding a drop of ferric chloride solution the deep purplish-red coloration characteristic of meconic acid will be produced. Meconic acid is, however, extracted with difficulty and imperfectly by agitating its acidulated aqueous solution with ether. Amylic alcohol is a far better solvent.

The detection of meconic acid in the above manner of course proves the presence of opium in the tincture. When this information alone is sought the paregoric may be diluted in a test-tube with proof-spirit till it is of a light yellow colour, and a drop or two of solution of ferric chloride then added. If opium be present, a more or less deep red coloration will be produced, owing to the formation of iron meconate. By comparing the depth of red colour with that given by a standard tincture a rough indication of the proportion of opium present can be obtained; but the amount of meconic acid in opium is too variable to allow of much stress being placed on the result obtained. It sometimes happens that paregoric is coloured with cochineal or contains a variety of tannin, in which case the coloration with ferric chloride becomes obscured. On cautiously adding hydrochloric acid, drop by drop, the colour produced by iron tannate is destroyed, while that due to the meconate persists till considerably more acid has been added.

The proportion of opium in paregoric is too small to allow of the ordinary method of determining morphine being conveniently used; but fair results, sufficiently accurate for most purposes, may be obtained by volumetric or colorimetric application of the reaction with iodic acid.

In addition to paregoric and its constituents, cough mixtures often contain other ingredients, among which may be mentioned oil of peppermint, squills, senega, horehound, and preparations of ipecacuanha. Oil of peppermint is readily recognised by the taste and odour. Squills are not readily recognised, the most hopeful plan being based upon the isolation and recognition of quillain. Senega is remarkable for the magnificent purple colour which it yields with strong sulphuric acid. The principle which gives the coloration is not extracted by amylic alcohol from either acid or alkaline solutions.

We are indebted to Mr. A. R. Tankard for valuable experiments on these and other points.

Ipecacuanha may be said to be almost a normal constituent of cough mixtures, and is of interest on account of the close resemblance of some of the colour-reactions of its alkaloids to those yielded by opium alkaloids. 
A remarkable case in this connection came within our experience a few months since. A cough mixture was sold which was expected to contain opium both as laudanum and as paregoric. The analyst to whom it was submitted reported it to contain morphine in a proportion of 0.12 per cent., or more than $\frac{1}{2}$ grain per ounce. A small portion of the same sample came into our hands, and we commenced its examination in the full expectation of confirming this result. Hence we submitted the mixture to a process adapted for the separation of morphine. For this purpose the sample was diluted with water, acidulated with hydrochloric acid, and the acid liquid treated with ether to remove the essential oils and any glucosides present. The acid liquid separated from the ether was then shaken with amylic alcohol, and that again separated in its turn. It was next neutralized with sodium bicarbonate and shaken with hot amylic alcohol to insure the extraction of any morphine present. The solution thus obtained was washed with water, and small portions evaporated for the application of colour-tests. The alkaloidal residue from the amylic alcohol gave only a doubtful reaction with ferric chloride,* but a very well. developed morphine reaction with Fröhde's reagent (sulphomolybdic acid), and an immediate reduction with iodic acid and a mixture of ferric chloride and ferricyanide of potassium. On the other hand, we could obtain no microscopic crystals of morphine, as may easily be obtained from such preparations containing morphine, by shaking out the amylic alcohol solution with a little dilute acetic acid, placing a few drops of the acetate solution in a watch-glass, or on a celled microscope-slide, and covering it with a watch-glass moistened with strong ammonia, so that the vapour may act on the liquid in the lower glass. After allowing it to stand for half an hour, the liquid is examined under the microscope, when the elongated prisms characteristic of morphine are readily detected. In this case, however, we failed wholly to obtain the long prismatic crystals characteristic of morphine, but obtained crystals of an entirely different kind, some of which were octahedra, closely resembling arsenious oxide, whilst others suggested the appearance of rice-starch.

At this stage our attention was called to the fact that, at an examination in analytical chemistry held last January, the candidates were required to analyse a liquid extract of ipecacuanha. Of the six who examined the extract several reported it to contain morphine. We have personally questioned three of these gentlemen, and find that they estimated the alkaloid by extracting it from an alkaline solution with amylic alcohol, and reported the presence of morphine as the result of several more or less satisfactory colour reactions. One of the candidates has stated in evidence the facts relating to this examination. Of course, by suitable means the ipecacuanha alkaloids could have been differentiated and separated from any morphine present, but the point is that, when a process suitable for isolation and determination of morphine was used, an alkaloidal residue was obtained which simulated morphine so as to be mistaken for that alkaloid. We consequently prepared the mixed alkaloids from several samples of the liquid extract of ipecacuanha by the same method which we had employed for examining the cough mixture in

* This result we attribute to the fact that we worked on a very limited amount of material. Both opium and ipecacuanha alkaloids give the well-known coloration, but the reaction is not nearly so delicate as that with Fröhde's reagent. 
question. We found the alkaloidal residue to give a well-marked greenish-blue coloration with neutral ferric chloride; a crimson changing to purple and blue colour with Fröhde's reagent; an immediate blue coloration with iodic acid and starch (with certain samples, but not with all); and an immediate formation of Prussian blue from a mixture of ferric chloride and potassium ferricyanide. The microscopic crystals obtained were, however, exactly similar to those which had been prepared from the cough mixture, and in no way resembled morphine. The crystalline appearance was so characteristic that we had no hesitation in reporting that the cough mixture contained ipecacuanha, and, in view of the colour reactions which extract of ipecasuanha of undoubted purity had given, we could find no evidence of the presence of morphine. We had searched for meconic acid, with wholly negative results, by the method which we had found serviceable in other cases-namely, extraction of the acidified liquid with amylic alcohol, evaporation of the solution, and testing the residue with ferric chloride.

After reporting the presence of ipecacuanha, the person who compounded the cough mixture informed us that he had actually introduced ipecacuanha wine into the cough mixture in question, but had not made any addition of a preparation of opium. It appeared evident, therefore, that the analyst who originally examined the mixture had mistaken the ipecacuanha alkaloids present for morphine.

The case affords an interesting example of the danger of relying on colourindications for alkaloids without a definite assurance that no interfering body is present. The remarkable manner in which the alkaloids of ipecacuanha similate those of opium in some of their reactions is not generally known, and we have felt it to be of such practical importance as to warrant us in bringing the matter before the Society in the detailed manner we have done.

\section{Discussion.}

Mr. William Chattaway stated that Mr. Allen had afforded him the opportunity of examining a portion of the original cough mixture, the composition of which had been such a vexed question, and he was able to confirm the authors' statements in every respect. The cough mixture gave several of the colour-reactions of morphine, but Fröhde's reagent, followed by hydrochloric acid, gave the magnificent Prussian blue coloration which had recently been found so characteristic of cephaëline. He also confirmed the authors' statements with respect to the characteristic crystals from ipecacuanha which they attributed to psychotrine. There was no difficulty in obtaining from the sample of cough mixture in question numerous microscopic crystals of this kind, and they were quite distinct in character from crystals of morphine obtained in an analogous manner.

Mr. Allen said that he was glad to know that his experience in this matter was so fully confirmed by that of Mr. Chattaway. There was no reflection on anyone who had made a mistake in the past when these reactions were not recognised, but care in the future was obviously necessary lest a preparation containing ipecacuanha might be mistaken for one containing the alkaloids of opium, especially as these colour-tests were much less definite and striking with the mixed 
alkaloids of opium than with pure morphine. The remarkable reaction for cephaëline with Fröhde's reagent and hydrochloric acid was of great assistance, but it was conceivable that the reaction with Fröhde's reagent might be complicated by the presence of other substances, and he thought that the most definite and characteristic evidence of the presence of ipecacuanha was really that afforded by the production of crystals of psychotrine.

Mr. Moor mentioned that with regard to tinct. camph. co. a standard had been recently issued by the Irish Local Government Board. That standard did not render it necessary to go very deeply into the question, but simply laid down the specific gravity, the alcoholic strength ( 57 per cent. by volume), and the total solids, which were put at 0.33 per cent. He was inclined to think, however, that that percentage of total solids was somewhat too high, and would like to hear Mr. Allen's opinion on this point.

Mr. Allen said that he was afraid he could not, without reference, give any figure for the total solids in paregoric. It was to be borne in mind, however, that at present this preparation was directed to be made, not with opium, of which the quantity used to be 40 grains per pint, but with an equivalent quantity of tincture of opium. The solids of the paregoric would therefore consist of the soluble matter of the opium from which the tincture was prepared, assuming all the other constituents of the paregoric to be volatile; so that from a knowledge of the average amount of solid matter contained in the laudanum-which, however, was liable to some variation-it might be ascertained by calculation whether the standard was correct.

Mr. Moor said that the quantity of tincture of opium directed to be taken was 60.9 c.c. per litre, and, assuming the average solids of the tincture to be 4 per cent., or a minimum of 3 per cent., the total solids of the paregoric might fall as low as 0.18 per cent., assuming the benzoic acid and oil of anise to be entirely volatile; and, as a matter of fact, he had figures of his own showing as little as 0.2 per cent. in a number of samples which he believed to be quite genuine. He mentioned this because the standard referred to was intended to form the basis of prosecutions; and if that were insisted upon, any sample falling below 0.33 per cent. in total solids would be prosecuted on. He thought, however, that it was not quite correct to place the standard so high.

Mr. R. A. CRIPPS said that he could quite confirm Mr. Moor's remark that paregoric might be perfectly genuine, and yet contain not more than 0.2 per cent. of total solids. In the examination of cough mixture, it seemed to him that ipecacuanha was almost the first thing to be expected; and with the knowledge that the alkaloids of ipecacuanha were soluble in amylic alcohol, a reasonable course would seem to be to extract first in the alkaline condition with ether, which would remove the cephaëline and emetine and leave the morphine.

Mr. ALLEN said that psychotrine would also remain undissolved after such a process as that suggested by Mr. Cripps, and psychotrine was the alkaloid which gave the characteristic crystals he had described. Chloroform would be preferable, but he would be very shy of treating a small quantity of such a preparation as a cough mixture with ether or chloroform for the purpose of removing one class of 
alkaloids and leaving the other. As a matter of fact, however, his purpose had been rather to describe the way in which the matter had actually been dealt with in the past than to suggest what might be the best way of dealing with it.

Mr. CRIPPs said that, as far as his experience went, there was no practical difficulty in the treatment with ether for removing the alkaloids other than morphine. 